\title{
Spatial updating of multiple targets: Comparison of younger and older adults
}

\author{
Christopher R. Bennett ${ }^{1,2}$ • Jack M. Loomis ${ }^{3}$ - Roberta L. Klatzky ${ }^{4}$. \\ Nicholas A. Giudice ${ }^{2,5}$
}

Published online: 26 June 2017

(C) Psychonomic Society, Inc. 2017

\begin{abstract}
When walking without vision, people mentally keep track of the directions and distances of previously viewed objects, a process called spatial updating. The current experiment indicates that while people across a large age range are able to update multiple targets in memory without perceptual support, aging negatively affects accuracy, precision, and decision time. Participants (20 to 80 years of age) viewed one, three, or six targets (colored lights) on the floor of a dimly lit room. Then, without vision, they walked to a target designated by color, either directly or indirectly (via a forward turning point). The younger adults' final stopping points were both accurate (near target) and precise (narrowly dispersed), but updating performance did degrade slightly with the number of targets. Older adults' performance was consistently worse than the younger group, but the lack of interaction between age and memory load indicates that the effect of age on performance was not further exacerbated by a greater number of targets. The number of targets also significantly increased the latency required to turn toward the designated target for both age groups. Taken together, results extend previous work
\end{abstract}

Nicholas A. Giudice

nicholas.giudice@maine.edu

1 The Laboratory for Visual Neuroplasticity, Department of Ophthalmology, Massachusetts Eye and Ear Infirmary, Harvard Medical School, Boston, MA, USA

2 Virtual Environments and Multimodal Interaction (VEMI) Laboratory, The University of Maine, Orono, ME, USA

3 Department of Psychological and Brain Sciences, University of California, Santa Barbara, CA, USA

4 Department of Psychology, Carnegie Mellon University, Pittsburgh, PA, USA

5 Spatial Informatics Program, School of Computing and Information Science, The University of Maine, Orono, ME, USA showing impressive updating performance by younger adults, with novel findings showing that older adults manifest small but consistent degradation of updating performance of multitarget arrays.

Keywords Aging · Spatial memory · Visual perception · Spatial updating $\cdot$ Spatial image

As people walk through the environment, the distances and directions to objects and landmark features are continually changing. Humans exhibit a remarkable ability to keep track of these spatial relations without having to keep the surroundings in view, a process known as spatial updating. Little research, however, has addressed how this ability changes with age, and how aging effects might be modulated by factors that affect spatial updating. The present study addresses such questions and reports impressive accuracy and precision for spatial updating that only diminished slightly in younger adults (1836 years) as the number of locations to be monitored was varied from one to six. Older adults (60-76 years) performed more poorly than the younger adults in terms of updating accuracy and precision. However, manipulations of target number that affected updating performance had comparable effects on both groups, pointing to a similarity in underlying spatial processing between groups, albeit with degraded overall effectiveness by older adults.

Theories of spatial updating have differentiated two general models: allocentric and egocentric. By the allocentric model, all locations, including that of a navigator, are designated in terms of extrinsic coordinates, and the navigator's position is updated over the course of movement. By the egocentric mod$\mathrm{el}$, the origin is centered on the navigator, and external locations are updated relative to that origin as the navigator moves. We adopt here the egocentric model for spatial updating 
during locomotion, given the compelling body of support (Giudice, Klatzky, Bennett, \& Loomis, 2013; Loomis, da Silva, Fujita, \& Fukusima, 1992; Wang et al., 2006; Wolbers, Hegarty, Büchel, \& Loomis, 2008). The core process of the model is path integration: moment-to-moment revision of location-to-self coordinates during locomotion. We assume, following the model of Byrne, Becker, and Burgess (2007), that updating the coordinates of a navigator-centered location requires that it be currently represented in the navigator's working memory. Additional processes needed to support updating would include encoding locations into memory, adding new locations to an existing representation as needed, and generating responses.

We have referred to the working-memory representation of spatial layout that is subject to updating as the spatial image (Loomis, Klatzky, \& Giudice, 2013; Loomis, Lippa, Klatzky, \& Golledge, 2002). As reviewed by Loomis et al. (2013), a key empirically supported property of the spatial image is that it constitutes a three-dimensional representation of locations in the surrounding environment. Note that locations are defined by their spatial parameters and need not be associated with, nor differentiated by, specific object identities. Spatial images are capable of being developed from multiple input modalities but are not necessarily veridical with the physical world, as they inherit systematic error associated with their perceptual origins (e.g., underperception of the distance to a sound source). In addition, locations represented in a spatial image are postulated as being stable and externalized relative to the observer.

As the spatial image is a working memory representation, it is subject to the well-known capacity limitations of transient storage. We assume that each location imposes a load on working memory. If the number of locations represented in the spatial image exceeds its capacity, more enduring longterm spatial memory can be called into play to handle the overload. In this case, further processing would be needed to retrieve locations from long-term memory, thereby reinstantiating them in the spatial image, which could require conversion from allocentric parameters (constituting a "cognitive map") to the current egocentric representation (Amorim, Glasauer, Corpinot, \& Berthoz, 1997).

To the extent that the load from representing locations in the spatial image falls within the limited capacity available in working memory, updating performance could be independent of the number of locations that are being monitored. This prediction relies, however, on the strong assumption of a discontinuity between processes that stay within, versus cross, the capacity line, rather than a continuously graded load effect. Spatial updating research has in fact shown mixed results with respect to the effect of memory load, depending on factors such as target exposure, updating type (translation versus rotation), and varying set size. The present research addresses the connections between memory load and spatial updating in a paradigm that probes these effects as a function of age-related changes.
A motivation for the current work was a seminal study by Rieser and Rider (1991, Experiments 2 and 3), which employed a blindfolded point-to-target task. Participants were visually exposed to one to five objects at predefined target locations. They then pointed without vision to a designated object, either from the origin directly after learning or after walking along a path with one or more turns (guided by an experimenter) to a novel test location. The latter requires spatial updating. Signed error and variable error $(S D$ of signed error) were reported as invariant over the number of targets with both children and young adults. Two other studies with adults under 55 years of age have also shown no effect of the number of targets on spatial updating performance within the range of one to four targets. Wolbers and colleagues (2008) compared performance in a pointing task after static viewing versus after a simulated forward translation, which requires updating. For error and latency measures, the additional effect of updating was invariant across load. Similarly, Loomis and colleagues (1998) presented one or two targets in an open field and found no load effect on participants' report of a designated target location. This held over variations in presentation modality (visual or auditory), form of report (verbal or walking), and whether participants reported from the origin or after walking forward $5 \mathrm{~m}$. Still, larger numbers of targets have been investigated in tasks requiring updating during rotation only (i.e., no translation), with somewhat equivocal results. It is interesting to note that the capacity to update under pure rotation, as measured by pointing latency and error, has been found to be insensitive to the number of spatial targets up to fairly large set sizes that likely exceed the span of spatial working memory (e.g., eight in Harrison, 2007; 15 in Hodgson \& Waller, 2006). However, spatial updating during rotation is computationally less demanding than updating during translation. Because the distance to a target has no effect on its change in direction, all targets undergo the same change in direction (equal to the head rotation), making the updating computations during rotation much simpler.

In contrast to the previous studies, some research has also found memory load effects on spatial updating performance. Wang and colleagues (2006) argued that if the process of updating is done allocentrically, then it will not suffer from set size increases, because an allocentric process requires updating of only the navigator's location with respect to the environmental coordinates, whereas egocentric updating at higher set sizes requires updating all locations relative to the navigator. In their study, participants used a virtual environment to learn and update one to three targets. Updating was done by following a path to a new location that involved both translation and an updated heading at the final stopping point. Greater heading rotations and increased memory loads significantly reduced updating performance, which was taken as evidence for egocentric updating. 
Given that spatial updating is a fundamental component of navigation, it is noteworthy that relatively little research has examined performance across the life span. The present research contributes to bridging this gap, by comparing the ability of younger and older adults to perform spatial updating and path integration at various working-memory loads. In general, the literature with older adults in the spatial domain has found that many aging effects are task-specific (for reviews, see Klencklen, Després, \& Dufour, 2012; Moffat, 2009; Techentin, Voyer, \& Voyer, 2014). Given this variation, it is important to consider how age might affect the processes involved in the spatial-updating task. We summarize the component processes as (a) encoding and retaining spatial locations in working memory, (b) path integration and spatial updating, and (c) generating and executing locomotor responses to a target. These processes will be dealt with in turn in the following paragraphs.

Research with older adults in the spatial domain provides mixed evidence for the preservation of the process of encoding and retaining spatial locations across much of the life span. In general, working memory shows an age-related decline that follows a linear trend as a function of age (Moffat, 2009; Park, 2000; Park et al. 2002). However, some studies have found that while allocentric spatial memory is most affected, egocentric spatial memory for locations tends to be preserved with age (Antonova et al., 2009; Desrocher \& Smith, 1998). The preservation of egocentric spatial memory has even been demonstrated at set sizes near assumed capacity limits (six targets in Olson et al., 2004). Mixed results have been found for age effects when learning locations from maps and small-scale arrays not requiring physical movement to explore. While some have found clear evidence of spatial decline (Iachini, Iavarone, Senese, Ruotolo, \& Ruggiero, 2009; Kirasic, 2000; Moffat \& Resnick, 2002), others have shown preservation when memory prompts are present (Yamamoto \& DeGirolamo, 2012) or a mix of preservation and decline depending on alignment of perspective (Borella, Meneghetti, Muffato, \& De Beni, 2015). The encoding and retention of spatial layouts by older adults clearly merits further examination, as in the present studies. Given the evidence in the literature for general age-related memory changes and apparently limited preservation of spatial abilities, differences between the age groups are predicted in measures of encoding and retention.

Considering the process of spatial updating, we are not aware of extant research studying the updating of visually learned target arrays with an older adult sample. Perhaps most relevant are data from triangle completion tasks, which require updating the position of a starting point while walking a twoleg path without vision. Results have shown consistent decreases in accuracy or performance time with age (Allen, Kirasic, Rashotte, \& Haun, 2004; Adamo, Briceño, Sindone, Alexander, \& Moffat, 2012; Harris \& Wolbers, 2012;
Mahmood, Adamo, Briceño, \& Moffat, 2009). A study by the present authors investigated updating locations in fourtarget, table-top arrays encoded through touch, across the life span. Results showed that adults over 60 years of age produced significantly more errors after updating than did younger adults (Giudice, Bennett, Klatzky, \& Loomis, 2017). Older adults have shown dramatic increases in both errors and response time in various other tasks requiring relatively complex processing of spatial representations, for instance, mental rotation (Hertzog, Vernon, \& Rypma, 1993) and spatial navigation through virtual mazes (Rodgers, Sindone, \& Moffat, 2012; Zakzanis, Quintin, Graham, \& Mraz, 2009; for reviews, see Moffat, 2009; Klencklen et al., 2012). Based on these results, we expect to observe age-related declines in measures of updating ability in the current study.

Finally, we consider the processes of generating responses and executing actions. If the process of generating a response from a memory representation is akin to a cognitive decision, one could expect the time to initiate locomotion after updating to increase with age (Moffat, 2009; Park, 2000). Of course, locomotor performance would also be expected to be slower and potentially more imprecise due to aging effects on motor abilities; caution could also play a role (Buzzi, Stergiou, Kurz, Hageman, \& Heidel, 2003; Rosano et al., 2012). Mobility plays an important role in executing the physical demands of spatial tasks. Links have been made between mobility and cognitive performance, including spatial tasks such as mental rotation (Jansen \& Kaltner, 2014). These effects would emerge in analyses of distance error, both random (precision) and systematic (bias), which we investigated here.

To summarize, a number of attempts have been made to assess the process of spatial updating and how it is affected by the complexity of spatial layout. In general, the method first exposes participants to some number of locations and then instructs them to update these locations. The current study extends this earlier work to a larger target size (six), introduces methodological changes to isolate spatial updating processes in working memory, incorporates multiple process-specific measures, and, above all, examines differences between younger (18 to 36 years) and older (60 to 76 years) age groups. The isolation of spatial updating by directed measures is particularly important given the range of processes that could be age dependent.

Participants viewed spatial layouts of one to six targets from an initial location. Following learning, they were asked to walk blindfolded to one of the target locations, designated either in advance of (or during) locomotion. By limiting the arrays to six items or fewer, it was our goal to enable participants across the life span to maintain the presented array in working memory without transfer to long-term memory. We also discouraged transfer of arrays to long-term memory by testing immediately after learning and varying the target array from trial to trial. High computational demands on spatial 
updating were enforced by having participants translate as well as rotate. Repeated observations using balanced layouts allowed us to collect data from a single participant approaching the same target location in all experimental conditions. Multiple dependent variables collected in both age groups were used to assess effects of aging on different components of the task. Specifically, the time to encode the target array; accuracy and precision of walking toward a designated target, directly or indirectly; and decision time were measured at different memory loads. With this range of methods, key manipulations, and targeted measures, the present work aimed to address the basic question of whether older adults successfully encode and update spatial representations at different levels of complexity, and how their performance reflects the processes of aging.

\section{Method}

Participants A total of 40 participants participated in the experiment. They comprised two groups of equal size, with the younger adult group (10 females) ranging from ages 18 to 36 $(M=23.5, S D=4.5)$ and the older adult group (12 females) ranging from ages 60 to $76(M=68.5, S D=4.6)$. Due to the sample size, the power to detect small effects is limited. Participants were closely matched for years of educational experience, with the older adult group averaging 16.5 years and the younger adult group just under 16 years (with 16 representing an undergraduate degree). To screen for color deficiency, participants had to twice identify the color of each of the six targets in a randomly presented order prior to beginning the experiment (everybody passed this test). To screen for cognitive impairment, the Short Blessed Test was used, with all participants scoring less than 4 (scores above 4 indicate possible cognitive impairment; Carpenter et al., 2011). The University of Maine's local ethics committee approved the research, and written informed consent was obtained from all participants, who received monetary compensation for their time.

Apparatus The stimuli used for this study consisted of six 7.6-cm diameter battery powered, tap-on LED light targets (Sylvania Tap Light Model \#36010). Each light was covered with a different colored filter, created by wrapping craft-grade cellophane over the LED, to create red, blue, green, yellow, orange, and purple targets. A pilot study was conducted to ensure that the lights were balanced in terms of brightness. Wireless headphones (Creative Labs, HS-1200) were worn during the study to deliver the auditory color target labels and to provide white noise between trials to mask the target placement by the experimenter. The color names were delivered using AT\&T demo text-to-speech software (AT\&T Labs, Florham Park, NJ). A blindfold (Mindfold Inc., Tucson, AZ) was worn between all experimental trials to eliminate the possibility of the participant seeing the target setup. An infrared LED mounted on the top of the headphones was used for tracking user movement in the $6 \mathrm{~m} \times 6 \mathrm{~m}$ lab room during the blind walking tests by means of a four-camera optical tracking system (Model PPT, Worldviz Inc., Santa Barbara, CA). Recording of tracking data and sequencing of experimental trials was done using the Vizard 3D rendering suite (Version 3.17, Worldviz). A Nintendo Wii-mote was used for making keypress responses during the blind walking trials.

Design and procedure On each trial, the participant was exposed to one, three, or six target locations, one of which was designated to be reached by walking either directly from the origin or via a turn point at $0.5 \mathrm{~m}$ or $1.0 \mathrm{~m}$ from the origin. The 12 locations of the targets were selected from combinations of two distances $(1.5 \mathrm{~m}$ and $2.25 \mathrm{~m})$ and six angles $\left(-60^{\circ},-40^{\circ}\right.$, $20^{\circ}, 20^{\circ}, 40^{\circ}$, and $60^{\circ}$ with respect to a work space north designated $0^{\circ}$; negative signs represent targets on the left). See Fig. 1 for a top-down view of the spatial layout.

The study used a mixed-model design, with the number of targets (one, three, or six) and walking turn point (direct walk, indirect walk of $0.5 \mathrm{~m}$, or indirect walk of $1.0 \mathrm{~m}$ ) constituting within-participants factors and age group as a betweenparticipants factor. Each participant performed a total of 27 trials. Of these, nine trials were directed to a critical location chosen a priori, such that each participant walked to that same point with all combinations of the number of targets and walking turn points. Two critical target locations were used, one at $40^{\circ}$ and $2.25 \mathrm{~m}$ and the other at $60^{\circ}$ and $1.5 \mathrm{~m}$. Participants

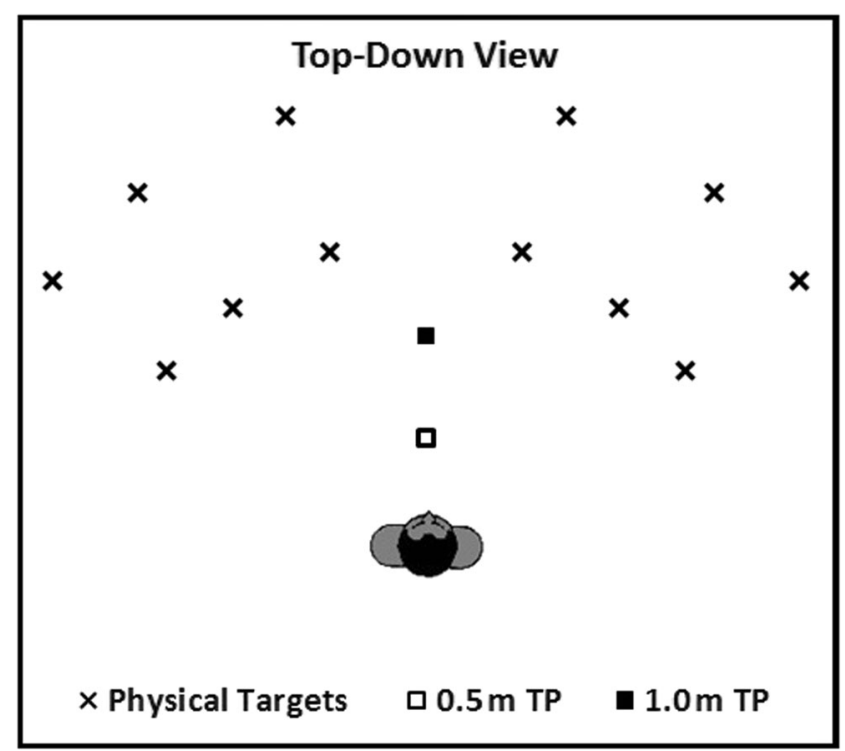

Fig. 1 Top-down view of the spatial layout. Each $\boldsymbol{X}$ represents a possible location for an object. The two squares represent the two turning points $(0.5 \mathrm{~m}$ and $1.0 \mathrm{~m})$ from the body showing the start location. From these 12 possible locations, 1,3 , or 6 targets for any given trial were chosen 
were alternately assigned to one of the critical points, which thus constituted a between-subjects variable. The 18 noncritical trials were balanced within participants, such that there were an equal number of trials with left and right targets, the number of targets presented, and turning points.

Each trial consisted of two phases, successively involving memory encoding and spatial updating. At the start of a trial, the participant stood blindfolded at the origin facing $0^{\circ}$ as oriented by a toe rest on the floor. Negative angles for target locations represent a counterclockwise rotation from the origin and positive angle values represent a clockwise rotation. Headphones were worn to mask ambient room sounds and to give instructions. After the experimenter silently placed the color target(s) for that trial and dimmed the room lighting, the participant was instructed to lift his or her blindfold in the now dimly lit room and observe the color and location of the target light(s). The memory-encoding period ended when the participant judged that the target locations had been accurately encoded or after $45 \mathrm{~s}$ had elapsed. Participants were informed about this time limit prior to the beginning of the experimental trials. This encoding duration was deemed appropriate given the use of verbal queuing of the targets and the requisite target/name association required to perform the testing task. A pilot study confirmed that this time was more than sufficient to encode up to six targets. Previous studies addressing multiple target arrays have employed similar self-paced learning (Hodgson \& Waller, 2006).

The updating phase began shortly after the memoryencoding phase. The participant lowered the blindfold, and the experimenter(s) silently removed the targets from the walking space and initiated the program for measuring the participant's trajectory. This process took approximately $5 \mathrm{sec}-$ onds. During the updating phase, participants either heard a click sound or a color name through the headphones (predetermined through a pseudorandom design). If a color was named, the participant's task was to walk directly to its location (direct walk condition), stopping once there and verbally indicating completion of the response. The experimenter pushed a button on the Wii-mote as soon as the verbal response was given, triggering a time stamp and recording the response location (done programmatically based on location of the LED on top of the headphones). If a click was heard (indirect walk condition), the participant's task was to walk directly forward until a color name was heard through the headphones (triggered programmatically by the tracking system described in the apparatus section). The color name was given at either the $0.5 \mathrm{~m}$ or $1.0 \mathrm{~m}$ turning points. Participants were neither informed of the exact location of the turning points prior to the experiment nor that the click sound was associated with two distances. Upon hearing the color, the participant was to immediately re-orient to face the target and walk directly to the requested location, again verbally indicating completion of the response once there. This procedure requires spatial updating, as participants were not aware of when they would be requested to reorient while they walked forward. After each response, the participant was guided back to the origin location by means of a short guidance rod via a straight line from their stopping point. Once back at the starting point, the next memory-encoding phase began.

Participants were also given practice trials that involved the same experimental procedure as the actual trials, but with only two targets positioned at locations not used in the experiment while performing direct walks. They received visual feedback (i.e., were allowed to see where they stopped in relation to where they started) only during these practice trials. After the experiment, participants were questioned whether they noticed the more frequent occurrence of the critical position; none reported affirmatively.

\section{Results and discussion}

Elimination of outliers For each measure, outliers were chosen based on 2.5 standard deviations above the mean for the given response condition (number of targets). Those outliers were then replaced with the group mean (not including the outlier data). Overall, $2.9 \%$ of the data was replaced for the older adults and $2.4 \%$ for the younger adults.

Method of analysis On all trials, participants performed spatial updating while walking to the encoded target location. However, on direct walking trials, because they were told the color of the target before beginning to walk, they never had to update more than a single target along the direct path of approach. For this reason, we report two types of analyses measuring the process of spatial updating. The first uses a variable (turn type) that averaged participants' data across the two indirect conditions $(0.5-\mathrm{m}$ turn and $1.0-\mathrm{m}$ turn) and then compared the pooled indirect trials to the direct trials. A preliminary omnibus mixed-model ANOVA, which indicated no significant effects of turn point or interactions between turn point and age group (all $p \mathrm{~s}>.50$ ), justified averaging across participants' responses for the two indirect points. This subsequent analysis employed a mixed-model ANOVA, with number of targets and turn type as within-participants factors and age group as a between-participants factor. The second analysis evaluates whether the number of targets influenced the performance of updating by using only the indirect walking trials (incorporating a turn point at 0.5 or $1.0 \mathrm{~m}$ ), where the memory load varied prior to the participants' being informed of the color of the target. This analysis also used a mixedmodel ANOVA, with number of targets and turn point as within-participants factors and age group as a betweenparticipants factor. Both analyses were run for accuracy, precision, and decision time. Learning time was run in a separate 
ANOVA, with only number of targets and age groups as the within- and between-participants factors, respectively.

Direct versus indirect: Accuracy This analysis contrasts trials where the target was specified in advance of walking, enabling updating of a single target along the path of approach, versus those where spatial updating of multiple targets was required before one was designated and incorporates a two-leg path. Age and number of targets are also factors. Figure 2 (older adults) and Fig. 3 (younger adults) show the centroids of the terminal points of the trajectories walked to the critical locations, averaged over participants, as a function of the number of targets and turn point. High accuracy is indicated by close proximity of the centroids to the targets.

Updating accuracy on a given trial was assessed in terms of the distance error (distance between a participant's terminal point and the corresponding target), which averaged $44 \mathrm{~cm}$ (41 cm for direct and $45 \mathrm{~cm}$ for indirect trials) for older adults, and $29 \mathrm{~cm}$ (24 cm for direct and $31 \mathrm{~cm}$ for indirect trials) for younger adults. The ANOVA revealed significant effects of both number of targets, $F(2,36)=5.24, p=.007, \eta_{\mathrm{p}}{ }^{2}=0.12$, and age group, $F(1,36)=24.83, p<.001, \eta_{\mathrm{p}}{ }^{2}=0.40$. Turn type was also significant, $F(1,36)=4.27, p=.046, \eta_{\mathrm{p}}{ }^{2}=0.10$, but there were no significant interactions. Post hoc Bonferroni tests for number of targets found significant differences between one versus six targets $(p=.008)$ and three versus six targets $(p=.018)$. The significant result of number of targets and age group suggests that six targets were more difficult to update than one, and younger adults overall outperformed the older adults. The lack of a reliable interaction between age group and number of targets, $F(4,72)=0.13, p=.874, \eta_{\mathrm{p}}{ }^{2}$ $<0.01$, indicates that the effect of age on accuracy was not further exacerbated by a greater number of targets. The significance of turn type (direct vs. indirect trials) reveals a tendency for the direct trials to produce more accurate responses, but, once again, the lack of any interaction with age group suggests that the impact of anticipating multiple targets during spatial updating was not greater for older adults.

Indirect trials only: Accuracy The most appropriate way of assessing whether number of targets influenced updating accuracy is to focus on only the indirect trials, for which the participant did not know the target location until reaching one of the two turn points. Again, accuracy was assessed in terms of distance error, the distance between a participant's terminal point, and the corresponding target distance. The ANOVA allowed us to examine the effect of age when updating multiple targets at varying distances was required. The results revealed significant effects of number of targets, $F(2,36)=5.18, p=.009, \eta_{\mathrm{p}}{ }^{2}=0.12$, and age group, $F(1,36)=$ $12.71, p=.001, \eta_{\mathrm{p}}{ }^{2}=0.25$. No interaction between age group and number of targets was found, $F(4,72)=0.56, p=.562$, $\eta_{\mathrm{p}}{ }^{2}=0.02$. Figure 4 (upper panel) summarizes the accuracy

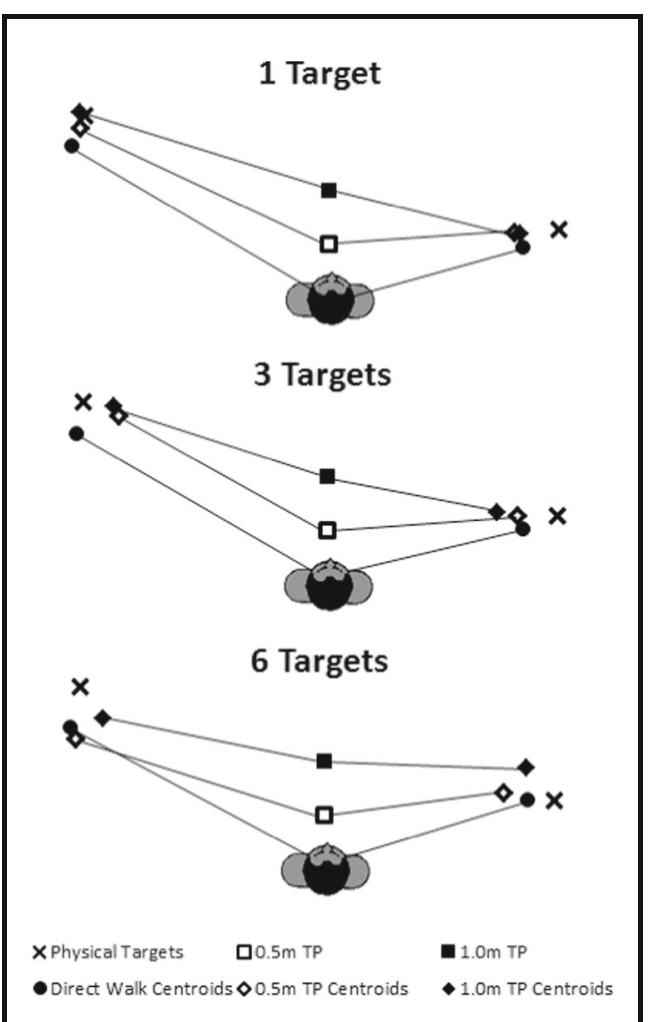

Fig. 2 Centroids of the terminal points for the older adult group, averaged over participants, of the walking trajectories to the critical locations as a function of the number of targets and turn point

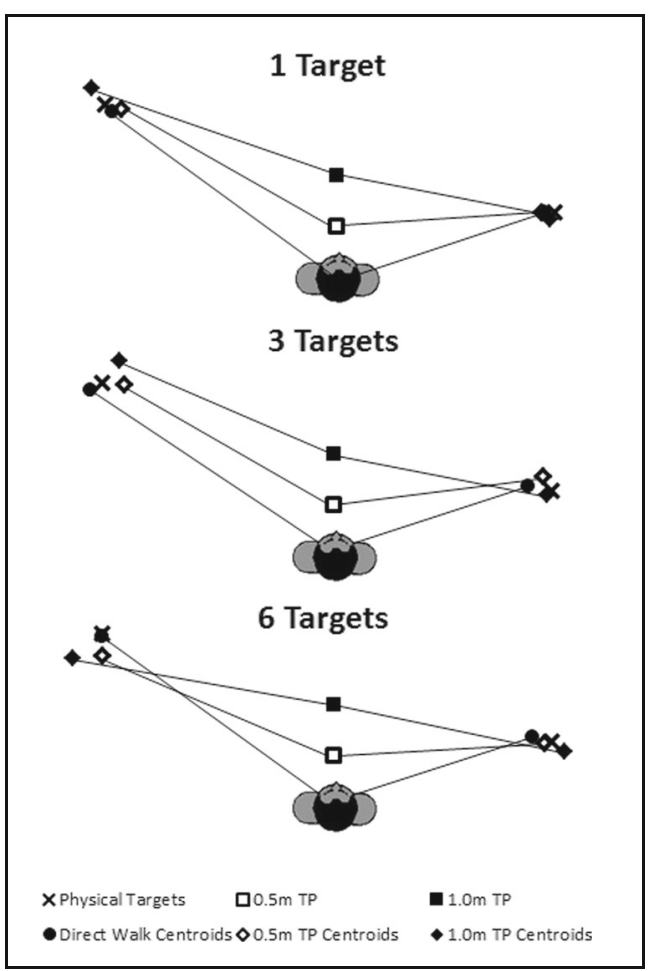

Fig. 3 Centroids of the terminal points for the younger adult group, averaged over participants, of the walking trajectories to the critical locations as a function of the number of targets and turn point 

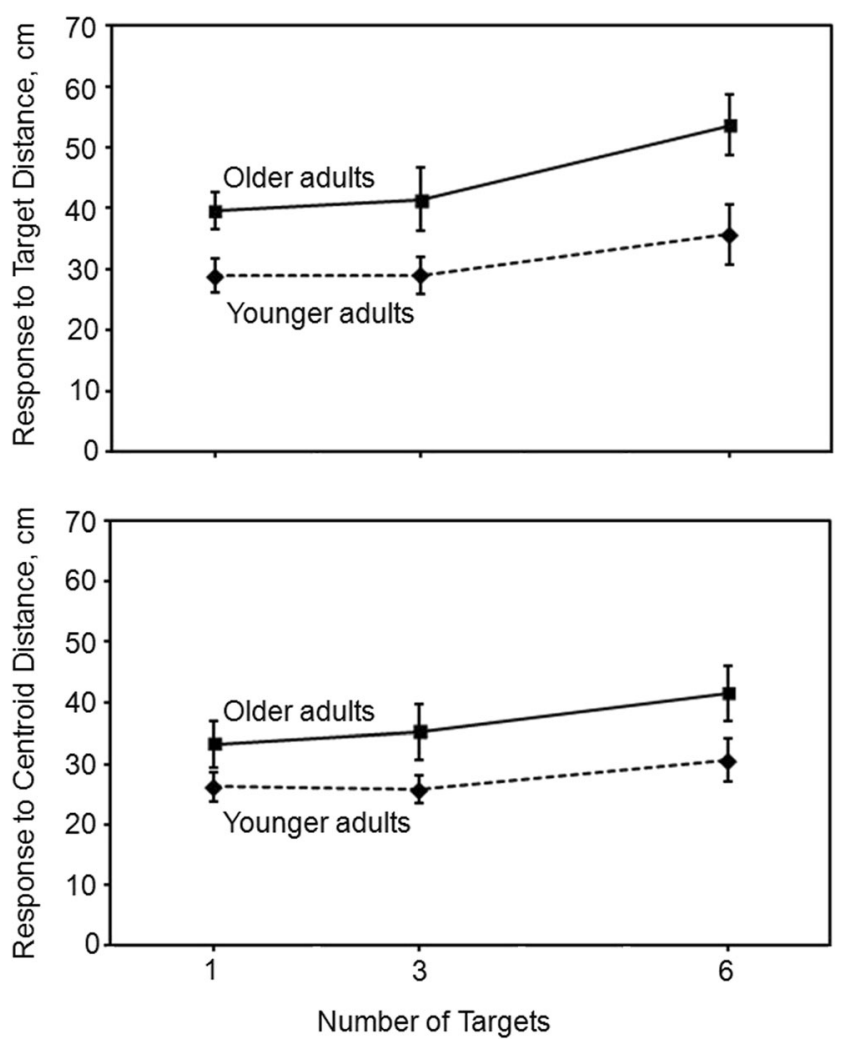

Fig. 4 Accuracy results (upper panel) and precision results (lower panel). Accuracy is indicated by the reciprocal measure of distance between the target and the participant's terminal location. Precision is indicated by the reciprocal measure of the distance between the group centroid and the participant's terminal location

results for the indirect trials, averaging over the turn points at 0.5 and 1.0 meters. The significant effects of number of targets and age on accuracy are apparent. The centroids for the indirect walks were very close to the respective targets for the younger adults, averaging just $14.3 \mathrm{~cm}$ away, while older adult centroids averaged $25.7 \mathrm{~cm}$ away.

Direct versus indirect: Precision Precision is the inverse of the spread of terminal points about the group centroid. Precision, in this regard, refers to the consistency of responses around the group-estimated perceived location and is taken as a measure of noise in the updating process, as contrasted with systematic error. This analysis examined the effects on precision of updating one (direct) versus multiple targets (indirect) varying in number. Figure 5 (older adults) and Fig. 6 (younger adults) show the terminal points of the individual walking trajectories relative to the group centroids (solid circles) as well as the targets (Xs) for just four of the nine conditions.

High precision is signified by tight clustering of the points about the centroid. In accord with this definition of precision, we computed the distance between the terminal point on each trial and the corresponding group centroid, which averaged $35 \mathrm{~cm} \mathrm{(31} \mathrm{cm} \mathrm{for} \mathrm{direct} \mathrm{and} 37 \mathrm{~cm}$ for indirect trials) for the older adult group and $26 \mathrm{~cm}(23 \mathrm{~cm}$ for direct and $28 \mathrm{~cm}$ for indirect trials) for the younger adults; a lower score means greater precision. The ANOVA on the precision measure revealed significant main effects for number of targets, $F(2,36)$ $=5.84, p=.004, \eta_{\mathrm{p}}^{2}=0.13$, age group, $F(1,36)=9.42, p=$ $.004, \eta_{\mathrm{p}}{ }^{2}=0.20$, and turn type, $F(1,36)=5.98, p=.019, \eta_{\mathrm{p}}{ }^{2}=$ 0.14 . No significant effects were found for any of the interactions, including age group and number of targets, $F(4,72)=$ $0.84, p=.434, \eta_{\mathrm{p}}{ }^{2}=0.02$. Post hoc Bonferroni tests revealed significant differences between one versus six targets $(p=$ $.023)$ and three versus six targets $(p=.045)$. These results demonstrate that both groups showed a dramatic reduction in their precision from one target up to six, and, once again, the younger adults showed better overall performance than their older counterparts. As with the accuracy analysis, the significance of turn type (direct vs. indirect trials) reveals a tendency for the direct trials to produce more precise responses, but the impact was not differential across age groups.

Indirect trials only: Precision As in the case of accuracy, the most appropriate way of assessing whether number of targets influenced updating precision is to focus on only the indirect trials, for which the participant did not know the target location until reaching one of the two turn points. As in the preceding comparison of direct and indirect trials, precision was assessed in terms of the distance between a participant's terminal point and the corresponding response centroid. The ANOVA on the precision measure included only indirect trials to examine effects of updating distance (turn point) and age. It showed significant effects only for age group, $F(1,36)=6.17$, $p=.018, \eta_{\mathrm{p}}{ }^{2}=0.14$. No interaction between age group and number of targets was found, $F(4,72)=0.20, p=.819, \eta_{\mathrm{p}}{ }^{2}=$ 0.01 . Because there was no effect of turn point and no significant interactions, Fig. 4 (lower panel) summarizes the precision results for the indirect trials only, averaging over the turn points at 0.5 and $1.0 \mathrm{~m}$. As with accuracy, the significant effects of number of targets and age on precision are apparent.

Direct versus indirect: Decision time For each response trajectory in the updating phase for critical targets, a decision time was calculated (see Fig. 7). This represents the elapsed time between the participant's hearing the target color and a $10^{\circ}$ change in the facing direction of their body toward the target, whether standing at the origin (direct trials) or at the turning points when walking indirectly.

This analysis compares decision times from when the target color was heard immediately before walking (direct) versus when participants first walked forward before target identification (indirect), by age and number of targets. Table 1 (left side) shows the results for the older age group, and Table 1 (right side) shows data for the younger adults. The ANOVA on decision time revealed significant results for number of targets, $F(2,36)=75.29, p<.001, \eta_{\mathrm{p}}{ }^{2}=0.67$, age group, $F(1,36)=4.57, p=.039, \eta_{\mathrm{p}}{ }^{2}=0.11$, and turn type (direct 


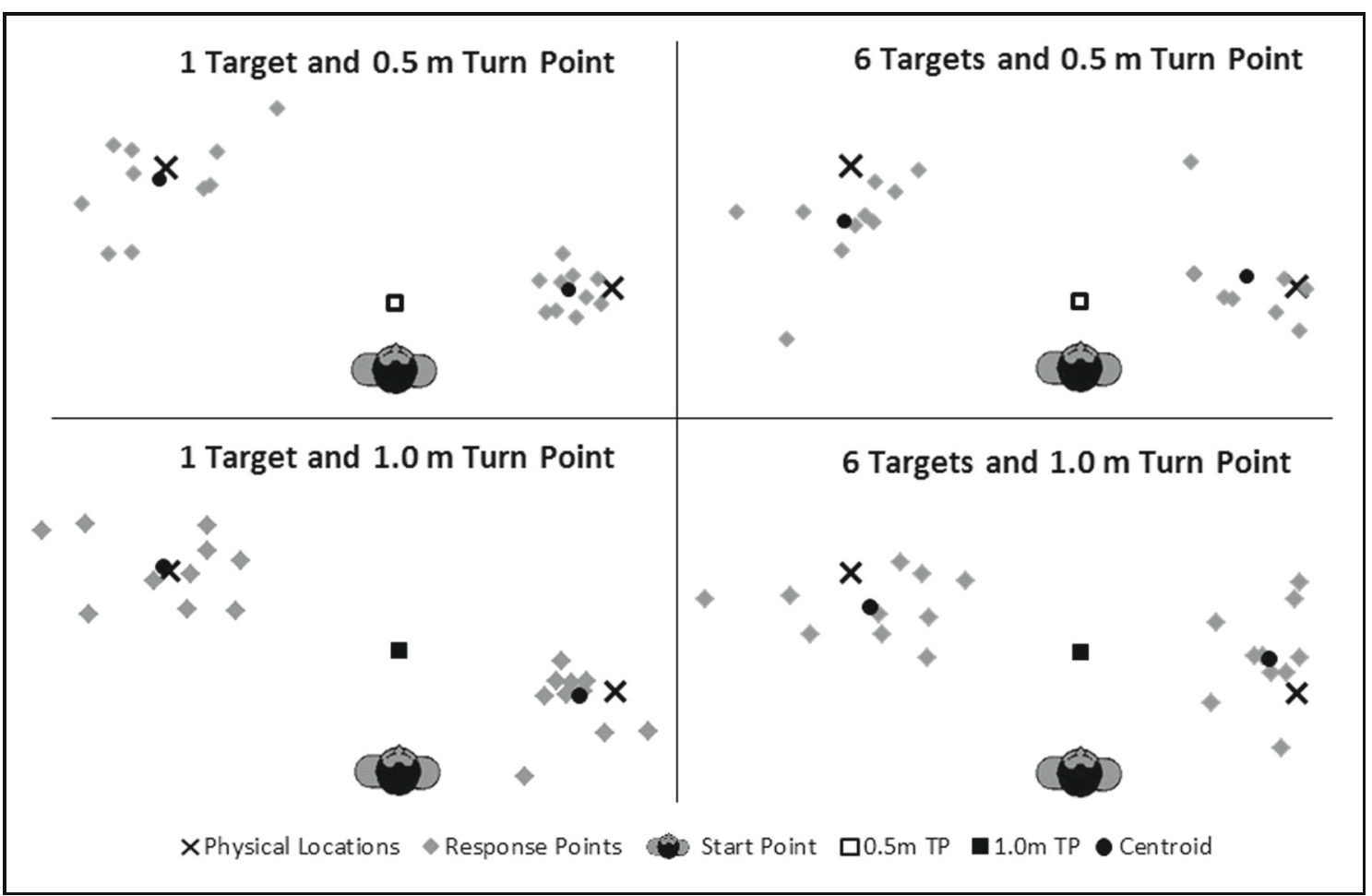

Fig. 5 Terminal points for the older adult group of individual walking trajectories relative to the group centroids (solid circles) as well as the targets $(X \mathrm{~s})$.

vs. indirect), $F(1,36)=34.29, p<.001, \eta_{\mathrm{p}}{ }^{2}=0.47$. There were also significant interactions between number of targets and age group, $F(4,72)=10.67, p=.001, \eta_{\mathrm{p}}{ }^{2}=0.22$, as well as between number of targets and turn type, $F(4,72)=7.61, p$
$=.003, \eta_{\mathrm{p}}{ }^{2}=0.17$. These results indicate that both age groups required additional time to decide on the location of the chosen target for an increasing number of targets. These findings also show that older adults required more overall decision

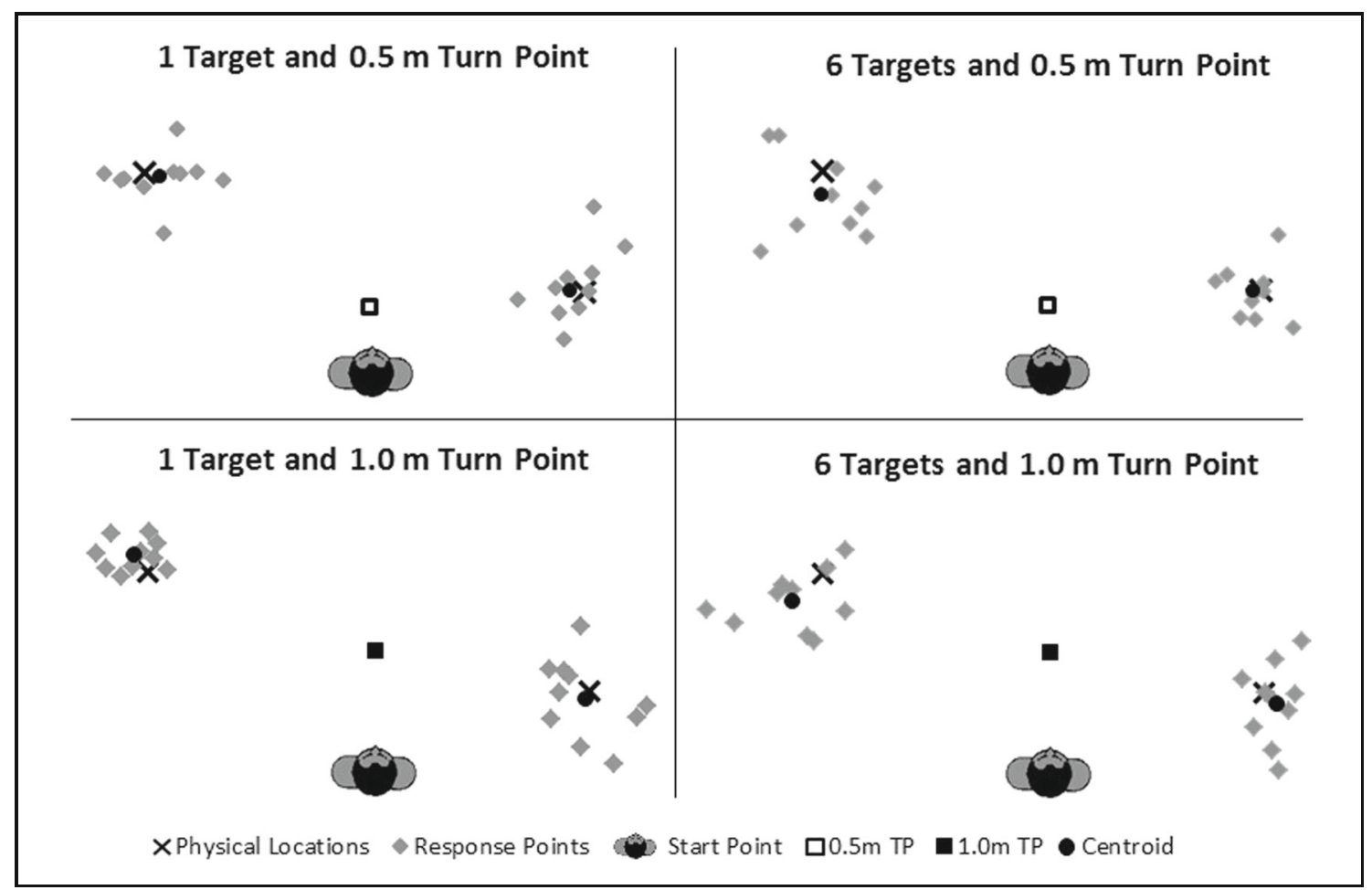

Fig. 6 Terminal points for the younger adult group of individual walking trajectories relative to the group centroids (solid circles) as well as the targets $(X \mathrm{~s})$. 


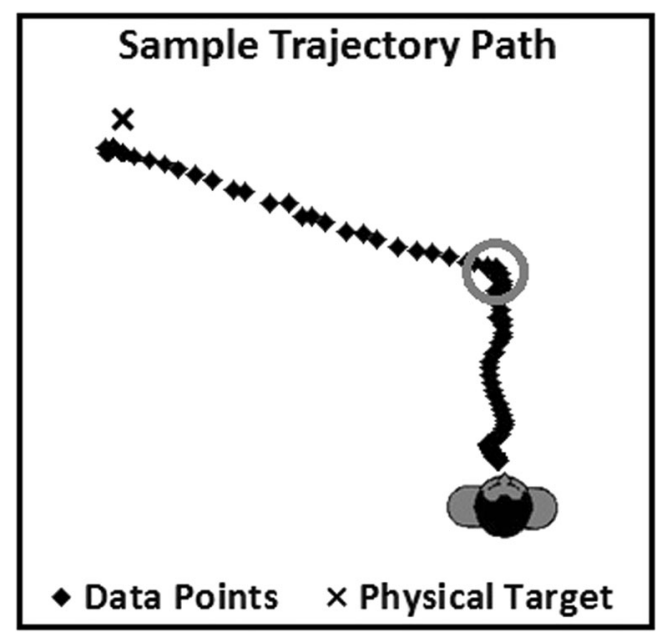

Fig. 7 Sample of trajectory path and turning point (circled region) used to calculate decision time, between the participant's hearing the target color and the turn, as defined by a $10^{\circ}$ change in his or her facing direction toward the target

time than the younger adult group, and that this disadvantage increased with number of targets.

Indirect trials only: Decision time This ANOVA separated the indirect walking data to isolate decisions when the target was not identified before walking, by number of targets and age group. There were significant effects for number of targets, $F(2,36)=35.57, p<.001, \eta_{\mathrm{p}}^{2}=0.48$, and age group, $F(1,36)=8.84, p=.005, \eta_{\mathrm{p}}{ }^{2}=0.19$. There was also a significant interaction, $F(4,72)=4.36, p=.037, \eta_{\mathrm{p}}^{2}=0.10$. Overall, the decision times for both age groups were affected by the number of targets, with the greatest latencies observed for sixtarget trials. The older adults also showed increased decision times as compared to the younger adults, most notably for the highest memory load (six targets). The increase in decision time shows that higher levels of memory load further impacted the decision making of the older adults.

Encoding time: All conditions This analysis examined time to encode the targets during initial learning. As the learning phase was done prior to participants' knowing whether the trial would be direct or indirect, an ANOVA pooling trials across this variable, with factors of number of targets and age, was performed. The results show significance for number of targets, $F(2,36)=278.99, p<.001, \eta_{\mathrm{p}}{ }^{2}=0.70$. Additionally, there was a significant interaction between number of targets and age group, $F(4,72)=3.61, p=.048, \eta_{\mathrm{p}}{ }^{2}=$ 0.03 . The significant effect of number of targets is simply a result of increasing the load on learning. Encoding time means for the older adult group increased from one target $(M=$ $6.79 \mathrm{~s}, S D=2.43)$, to three $(M=12.96 \mathrm{~s}, S D=5.31)$, to six $(M=26.83 \mathrm{~s}, S D=12.61)$. Encoding time increased for the younger adults from one target $(M=6.95 \mathrm{~s}, S D=2.78)$, to three $(M=12.79 \mathrm{~s}, S D=5.46)$, to six $(M=23.19 \mathrm{~s}, S D=$ 10.07). The interaction appears to reflect the small difference between the older and younger adults when load was highest (six targets). The times to learn lower set sizes were virtually identical across age groups. To test whether the longer learning time for older adults might compensate for other deficits, correlations were computed across participants between learning time and performance measures within each number of targets and age group; none were significant (all $p \mathrm{~s}>.5$ ).

Noncritical trial data As the noncritical target locations are not fully represented across all levels of the independent variables, there was potential for the data to be affected by location-specific error tendencies. Therefore, we do not report statistical analyses on accuracy and precision for noncritical locations. However, it is evident that under indirect as well as direct walking, participants converged on the targets with approximately the same level of accuracy and precision as was found for the critical locations. The distance of responses from the physical target locations (accuracy) for the noncritical trials of older adults were $47 \mathrm{~cm} \mathrm{(45} \mathrm{cm} \mathrm{for} \mathrm{direct} \mathrm{and} 48 \mathrm{~cm}$ for indirect trials) and $29 \mathrm{~cm} \mathrm{(27} \mathrm{cm} \mathrm{for} \mathrm{direct} \mathrm{and} 30 \mathrm{~cm}$ for indirect trials) for younger adults. The distance of responses from the respective centroid locations (precision) for the noncritical trials of older adults were $33 \mathrm{~cm} \mathrm{(28} \mathrm{cm} \mathrm{for} \mathrm{direct} \mathrm{and} 36 \mathrm{~cm}$ for indirect trials) and $26 \mathrm{~cm}(23 \mathrm{~cm}$ for direct and $27 \mathrm{~cm}$ for indirect trials) for younger adults. These data show that both accuracy and precision for the noncritical trials were within $3 \mathrm{~cm}$ of those for the critical trials.

Table 1 Decision time means (in seconds) for the critical target locations broken down by direct and indirect turning points. Left side contains older adult data while the right side contains the younger adult decision time means

\begin{tabular}{|c|c|c|c|c|c|c|}
\hline \multicolumn{4}{|c|}{ Older adult decision time means } & \multicolumn{3}{|c|}{ Younger adult decision time means } \\
\hline Targets & Direct $(S D)$ & Indirect $0.5 \mathrm{~m}(S D)$ & Indirect $1 \mathrm{~m}(S D)$ & Direct $(S D)$ & Indirect $0.5 \mathrm{~m}(S D)$ & Indirect $1 \mathrm{~m}(S D)$ \\
\hline 1 & $1.55(0.34)$ & $1.76(0.68)$ & $1.74(0.71)$ & $1.59(0.87)$ & $1.80(0.70)$ & $1.54(0.48)$ \\
\hline 3 & $2.13(0.81)$ & $2.60(0.89)$ & $2.53(0.94)$ & $1.72(0.66)$ & $1.94(0.60)$ & $1.82(0.61)$ \\
\hline 6 & $3.41(1.44)$ & $4.23(2.08)$ & $4.21(2.29)$ & $2.35(1.32)$ & $3.06(1.63)$ & $2.60(1.70)$ \\
\hline
\end{tabular}




\section{General discussion}

This article describes an experiment evaluating the relative ability of younger and older participants to update multiple locations during real-time body movement, inducing continuous changes in self-to-object directions and distances. In the introductory section, we described the processing components of this complex task as: encoding and retaining spatial locations in working memory, spatial updating (by path integration without vision), and generating and executing locomotor responses to a target. Our principal interest is in the process of spatial updating.

We incorporated multiple measures to assess these components. The accuracy in walking to the target (distance from target location) measures systematic error tendencies in updating. Although in principle, errors could result from encoding as well as updating, our controlled learning task and a limit of six targets were intended to isolate the updating process as a source of error. Variations in the precision of walking (deviations around response centroid) reflect the accumulated noise during the processes of updating. While, in principle, noise could accumulate during response execution, the response was held constant across the critical variable of number of locations in the layout. We also included direct measures of encoding time and the decision component of response generation.

The fundamental questions addressed in this experiment are as follows: (1) Can older adults update their spatial location relative to multiple targets while walking? (2) Does age affect updating performance? (3) If so, what components are affected? (4) Do variables intended to affect updating — number of targets and direct versus indirect walking (i.e., whether participants knew in advance of locomotion which target they were headed toward) - have greater impact on older adults? We consider each of these questions and the implications of the answers, in turn.

First, both age groups evidenced considerable ability to update their location relative to targets while walking. Overall, errors were small in relation to distances walked. Consider that targets were as far as $225 \mathrm{~cm}$ away from the origin, with the walking distances being somewhat longer for the indirect trials. Averaging over direct and indirect walking trials and number of targets, the responses of younger adults were just $29 \mathrm{~cm}$ distant from the physical target, while those of older adults were $44 \mathrm{~cm}$ distant, equating to distance errors on the order of $13 \%$ and $20 \%$ of maximum distance, respectively. The corresponding values for precision error (distance of terminal point from group centroid) were $12 \%$ for young adults and $16 \%$ for the older group, respectively. These findings supplement those of Rieser and Rider (1991), who demonstrated the ability of children and young adults to update from one to five targets in a more complex updating task. The smaller scale space used for this experiment may overestimate people's ability to update in terms of magnitude of error.
While we do not know whether the error as a percentage of target distance would change if the experiment was translated to a larger scale space, previous work has shown consistency of blind walking and distance estimation over larger scales (Loomis et al., 1992; Thompson et al., 2004). Future work with older adult populations is needed to further explore the similarities and differences between scale of the learned/ walked space and updating performance.

While both groups evidenced the ability to update self-tolocation coordinates during walking, age effects were observed on every measure: encoding, accuracy, precision, and decision time. The effects on encoding and decision time are to be expected given the literature on age-related declines in cognitive function reviewed in the introduction. As was noted there, the decision-time effect could also reflect caution in initiating the nonvisual walking response. For present concerns, the age effects in updating accuracy and precision are more critical, as aging effects in this domain have not been studied effectively. Accordingly, the present study presents novel new findings by demonstrating age-related declines in systematic updating error and noise. For quantification, relative to the younger age group, in the older group the distance error increased by $52 \%$, and the precision error increased by $35 \%$.

Notably, despite the finding that spatial updating ability declined with age, there was little evidence of an age difference in the effect of variables that impact on the updating process by loading spatial memory: Specifically, age did not show a significant interaction with number of targets in any of the analyses of accuracy and precision. Age also did not interact with the direct/indirect walking variable in analyses of either accuracy or precision. In contrast, age did show interactions with number of targets in analyses of the more general age-related measures of encoding time and decision time. The finding that age interacts with memory load in measures of encoding and decision, but not spatial updating, suggests that the age deficit in updating may reflect a relatively general decline in spatial processing rather than a specific deficiency in path integration. While it is clear that measures of updating were not spared in the effects of age, updating per se may be preserved beyond what examining those measures in isolation would indicate. This conclusion is reinforced by the finding that neither accuracy nor precision were affected by the distance of the unknown turning point introduced during the indirect walk trials. This result suggests that for both age groups, once spatial locations have been stored in working memory, the process of updating those locations under active locomotion does not erode the spatial representation.

Although the main concern of the present study was the effect of age on spatial updating, it also provides data of general interest to the field of spatial cognition. One set of findings relates to effects of memory load, in terms of number of spatial targets. As reviewed in the introduction, several previous studies have assessed the effect of number of targets held 
in memory during spatial updating. Notably, multiple studies with younger age groups have found no significant effect of memory load, extending beyond the typically assumed working memory span (Harrison, 2007; Hodgson \& Waller, 2006; Loomis et al., 1998; Rieser \& Rider, 1991; Wolbers et al., 2008). The present study departs from those in that we found a modest effect of memory load in young adults, although the loss in accuracy and precision was only observed at the largest set size $(N=6)$.

Note that on direct trials, as the participant knew the target identity before walking, the initiation of spatial updating had to be formulated only for that single designated location. Yet there was an effect of the number of targets on decision time on direct-walking trials. It is likely that this effect is due to the number of alternatives on choice reaction time (Hick, 1952, Hyman, 1953). It might also be argued that the decision-time effect reflects retrieval of information from long-term memory prior to updating. An additional explanation for this difference on direct trials could be due to hesitation related to declines in physical mobility for the older adult group, a possibility requiring additional research to disentangle. Previous literature has found impacts of motor execution on older adult task performance (Jansen \& Kaltner, 2014; Rosano et al., 2012). Given that all measures here show some age-related decrement, however, it seems unlikely that mobility alone provides an account. Future work should continue to parse out the encoding, updating, action planning, and motor processes, with the aim of quantifying the effect each imposes on older adults during spatial updating.

In sum, spatial updating is a complex task that has been little studied across the life span, despite its relevance to everyday activities. The findings in the current study showing decreased ability, yet preserved competency in patterns of spatial updating ability and memory-guided navigation are, we believe, a valuable addition to the research literature on aging. Older adults certainly maintain considerable ability to update objects they encounter, even for multiple target arrays. However, the clear evidence we observed of degraded performance with respect to their younger peers on the same task suggests that this critical navigational behavior exhibits notable age-related differences. We have argued, however, that the observed age effects may not be intrinsic to updating, so much as reflecting a more general decline in spatial processing that is revealed in the updating task. These results add to our understanding of age-related spatial abilities and contribute to an explanation of why navigation performance by this demographic is so often cited as being more error prone than their younger peers.

Acknowledgements This research was supported by NIH Grant R01EY016817. We thank Joshua Leger for programming assistance and Ashley Suitter and Rafael Ramos for assistance with running the study.

\section{References}

Adamo, D. E., Briceño, E. M., Sindone, J. A., Alexander, N. B., \& Moffat, S. D. (2012). Age differences in virtual environment and real world path integration. Frontiers in Aging Neuroscience, 4, 26.

Allen, G. L., Kirasic, K. C., Rashotte, M. A., \& Haun, D. B. M. (2004). Aging and path integration skill: Kinesthetic and vestibular contributions to wayfinding. Attention, Perception \& Psychophysics, 66, 170 .

Amorim, M. A., Glasauer, S., Corpinot, K., \& Berthoz, A. (1997). Updating an object's orientation and location during nonvisual navigation: A comparison between two processing modes. Attention, Perception \& Psychophysics, 59, 404-418.

Antonova, E., Parslow, D., Brammer, M., Dawson, G. R., Jackson, S. H. D., \& Morris, R. G. (2009). Age-related neural activity during allocentric spatial memory. Memory, 17, 125-143.

Borella, E., Meneghetti, C., Muffato, V., \& De Beni, R. (2015). Map learning and the alignment effect in young and older adults: How do they gain from having a map available while performing pointing tasks? Psychological Research, 79, 104-119.

Buzzi, U. H., Stergiou, N., Kurz, M. J., Hageman, P. A., \& Heidel, J. (2003). Nonlinear dynamics indicates aging affects variability during gait. Clinical Biomechanics, 18, 435-443.

Byrne, P., Becker, S., \& Burgess, N. (2007). Remembering the past and imagining the future: A neural model of spatial memory and imagery. Psychological Review, 114, 340-375.

Carpenter, C. R., Bassett, E. R., Fischer, G. M., Shirshekan, J., Galvin, J. E., \& Morris, J. C. (2011). Four sensitive screening tools to detect cognitive dysfunction in geriatric emergency department patients: Brief Alzheimer's Screen, Short Blessed Test, Ottawa 3DY, and the Caregiver-Completed AD8. Academic Emergency Medicine, $18,374-384$.

Desrocher, M., \& Smith, M. L. (1998). Relative preservation of egocentric but not allocentric spatial memory in aging. Brain and Cognition, 37(1), 91-93.

Giudice, N. A., Klatzky, R. L., Bennett, C. R., \& Loomis, J. M. (2013). Combining locations from working memory and long-term memory into a common spatial image. Spatial Cognition \& Computation, 13(2), 103-128.

Giudice, N. A., Bennett, C. R., Klatzky, R. L., \& Loomis, J. M. (2017). Spatial updating of haptic arrays across the lifespan. Experimental Aging Research, 43, 274-290.

Harris, M. A., \& Wolbers, T. (2012). Ageing effects on path integration and landmark navigation. Hippocampus, 22, 1770-1780.

Harrison, A. M. (2007). Reversal of the alignment effect: Influence of visualization and spatial set size. Proceedings of the 29th Annual Conference of the Cognitive Science Society (pp. 341-346). Red Hook, NY: Curran Associates.

Hertzog, C., Vernon, M. C., \& Rypma, B. (1993). Age differences in mental rotation task performance: The influence of speed/accuracy tradeoffs. Journal of Gerontology, 48, 150-156.

Hick, W. E. (1952). On the rate of gain of information. Quarterly Journal of Experimental Psychology, 4, 11-26.

Hodgson, E., \& Waller, D. (2006). Lack of set size effects in spatial updating: Evidence for offline updating. Journal of Experimental Psychology: Learning, Memory, \& Cognition, 32, 854-866.

Hyman, R. (1953). Stimulus information as a determinant of reaction time. Journal of Experimental Psychology, 45, 188-196.

Iachini, T., Iavarone, A., Senese, V. P., Ruotolo, F., \& Ruggiero, G. (2009). Visuospatial memory in healthy elderly, AD and MCI: A review. Current Aging Science, 2, 43-59.

Jansen, P., \& Kaltner, S. (2014). Object-based and egocentric mental rotation performance in older adults: The importance of gender differences and motor ability. Aging, Neuropsychology, and Cognition, 21, 296-316. 
Kirasic, K. C. (2000). Age differences in adults' spatial abilities, learning environmental layout, and wayfinding behavior. Spatial Cognition and Computation, 2, 117-134.

Klencklen, G., Després, O., \& Dufour, A. (2012). What do we know about aging and spatial cognition? Reviews and perspectives. Ageing Research Reviews, 11, 123-135.

Loomis, J. M., Da Silva, J. A., Fujita, N., \& Fukusima, S. S. (1992). Visual space perception and visually directed action. Journal of Experimental Psychology: Human Perception and Performance, 18, 906.

Loomis, J. M., Klatzky, R. L., Philbeck, J. W., \& Golledge, R. G. (1998). Assessing auditory distance perception using perceptually directed action. Perception \& Psychophysics, 60, 966-980.

Loomis, J. M., Klatzky, R. L., \& Giudice, N. A. (2013). Representing 3D space in working memory: Spatial images from vision, touch, hearing, and language. In S. Lacey \& R. Lawson (Eds.), Multisensory imagery: Theory \& applications (pp. 131-156). New York, NY: Springer.

Loomis, J. M., Lippa, Y., Klatzky, R. L., \& Golledge, R. G. (2002). Spatial updating of locations specified by 3-D sound and spatial language. Journal of Experimental Psychology: Learning, Memory, and Cognition, 28, 335.

Mahmood, O., Adamo, D., Briceño, E., \& Moffat, S. D. (2009). Age differences in visual path integration. Behavioural Brain Research, $205,88-95$.

Moffat, S. D. (2009). Aging and spatial navigation: What do we know and where do we go? Neuropsychology Review, 19, 478-489.

Moffat, S. D., \& Resnick, S. M. (2002). Effects of age on virtual environment place navigation and allocentric cognitive mapping. Behavioral Neuroscience, 116, 851-859.

Olson, I. R., Zhang, J. X., Mitchell, K. J., Johnson, M. K., Bloise, S. M., \& Higgins, J. A. (2004). Preserved spatial memory over brief intervals in older adults. Psychology and Aging, 19, 310.

Park, D. C. (2000). The basic mechanisms accounting for age-related decline in cognitive function. Cognitive Aging: A Primer, 11, 3-19.
Park, D. C., Lautenschlager, G., Hedden, T., Davidson, N. S., Smith, A. D., \& Smith, P. K. (2002). Models of visuospatial and verbal memory across the adult life span. Psychology and Aging, 17, 299.

Rieser, J. J., \& Rider, E. (1991). Young children's spatial orientation with respect to multiple targets when walking without vision. Developmental Psychology, 27, 97-107.

Rodgers, M. K., Sindone, J. A., III, \& Moffat, S. D. (2012). Effects of age on navigation strategy. Neurobiology of Aging, 33, 15-22.

Rosano, C., Studenski, S. A., Aizenstein, H. J., Boudreau, R. M., Longstreth, W. T., \& Newman, A. B. (2012). Slower gait, slower information processing and smaller prefrontal area in older adults. Age and Ageing, 41, 58-64.

Techentin, C., Voyer, D., \& Voyer, S. D. (2014). Spatial abilities and aging: A meta-analysis. Experimental Aging Research, 40, 395 425.

Thompson, W. B., Willemsen, P., Gooch, A. A., Creem-Regehr, S. H., Loomis, J. M., \& Beall, A. C. (2004). Does the quality of the computer graphics matter when judging distances in visually immersive environments? Presence, 13, 560-571.

Wang, R. F., Crowell, J. A., Simons, D. J., Irwin, D. E., Kramer, A. F., Ambinder, M. S., ... \& Hsieh, B. B. (2006). Spatial updating relies on an egocentric representation of space: Effects of the number of objects. Psychonomic Bulletin \& Review, 13, 281-286.

Wolbers, T., Hegarty, M., Büchel, C., \& Loomis, J. M. (2008). Spatial updating: How the brain keeps track of changing object locations during observer motion. Nature Neuroscience, 11, 1223-1230.

Yamamoto, N., \& DeGirolamo, G. J. (2012). Differential effects of aging on spatial learning through exploratory navigation and map reading. Frontiers in Aging Neuroscience, 4, 14. doi:10.3389/fnagi.2012. 00014

Zakzanis, K. K., Quintin, G., Graham, S. J., \& Mraz, R. (2009). Age and dementia related differences in spatial navigation within an immersive virtual environment. Medical Science Monitor: International Medical Journal of Experimental and Clinical Research, 15, CR140-CR150. 